PROCEEDINGS OF THE

AMERICAN MATHEMATICAL SOCIETY

Volume 127, Number 5, Pages 1517-1524

S 0002-9939(99)04635-3

Article electronically published on January 29, 1999

\title{
RULES AND REALS
}

\author{
MARTIN GOLDSTERN AND MENACHEM KOJMAN
}

(Communicated by Carl Jockusch)

\begin{abstract}
A " $k$-rule" is a sequence $\vec{A}=\left(\left(A_{n}, B_{n}\right): n<\mathbb{N}\right)$ of pairwise disjoint sets $B_{n}$, each of cardinality $\leq k$ and subsets $A_{n} \subseteq B_{n}$. A subset $X \subseteq \mathbb{N}$ (a "real") follows a rule $\vec{A}$ if for infinitely many $n \in \bar{N}, X \cap B_{n}=A_{n}$. Two obvious cardinal invariants arise from this definition: the least number of reals needed to follow all $k$-rules, $\mathfrak{s}_{k}$, and the least number of $k$-rules with no real that follows all of them, $\mathfrak{r}_{k}$.

Call $\vec{A}$ a bounded rule if $\vec{A}$ is a $k$-rule for some $k$. Let $\mathfrak{r}_{\infty}$ be the least cardinality of a set of bounded rules with no real following all rules in the set.

We prove the following: $\mathfrak{r}_{\infty} \geq \max (\operatorname{cov}(\mathbb{K}), \operatorname{cov}(\mathbb{L}))$ and $\mathfrak{r}=\mathfrak{r}_{1} \geq \mathfrak{r}_{2}=\mathfrak{r}_{k}$ for all $k \geq 2$. However, in the Laver model, $\mathfrak{r}_{2}<\mathfrak{b}=\mathfrak{r}_{1}$.

An application of $\mathfrak{r}_{\infty}$ is in Section 3: we show that below $\mathfrak{r}_{\infty}$ one can find proper extensions of dense independent families which preserve a pre-assigned group of automorphisms. The original motivation for discovering rules was an attempt to construct a maximal homogeneous family over $\omega$. The consistency of such a family is still open.
\end{abstract}

\section{INTRODUCTION}

In the present paper we present new cardinal invariants which emerged in the authors' investigations of homogeneous families. These numbers have intrinsic interest for the theory of the continuum (in fact we regard it as surprising that those numbers have not been discovered earlier).

In Section 1 we discuss cardinal invariants related to " $k$-rules." A $k$-rule is a sequence $\vec{A}=\left(\left(A_{n}, B_{n}\right): n<\omega\right)$ of pairwise disjoint sets $B_{n}$, each of cardinality $\leq k$, and subsets $A_{n} \subseteq B_{n}$. A subset $X \subseteq \omega$ (a "real") follows a rule $\vec{A}$ if for infinitely many $n \in \omega, X \cap B_{n}=A_{n}$.

A rule $\vec{A}$ is bounded if it is a $k$-rule for some $k \in \omega$.

The obvious cardinal invariants related to rules are the following: the least number of reals needed to follow all $k$-rules, $\mathfrak{s}_{k}$, and the least number of $k$-rules with no real following all of them, $\mathfrak{r}_{k}$. Let $\mathfrak{r}_{\infty}$ be the least number of bounded rules with no real following all of them.

Received by the editors February 4, 1997 and, in revised form, July 16, 1997.

1991 Mathematics Subject Classification. Primary 03E35; Secondary 03E50, $20 B 27$.

Key words and phrases. Cardinal invariants of the continuum.

The first author is supported by an Erwin Schrödinger fellowship from the Austrian Science Foundation (FWF).

The second author was partially supported by NSF grant no. 9622579 .

(C)1999 American Mathematical Society 
We compare the $\mathfrak{r}_{k}$ 's and $\mathfrak{r}_{\infty}$ among themselves and to well known cardinal invariants: covering of category, covering of Lebesgue measure, $\mathfrak{r}, \mathfrak{b}, \mathfrak{d}$ and the evasion numbers $\mathfrak{e}_{k}$ which were studied by Blass and Brendle. We prove:

(a) $\max (\operatorname{cov}(\mathbb{K}), \operatorname{cov}(\mathbb{L})) \leq \mathfrak{r}_{\infty}$;

(b) $\mathfrak{r}=\mathfrak{r}_{1} \geq \mathfrak{r}_{2}=\mathfrak{r}_{k}$ for all $k \geq 2$;

(c) $\mathfrak{s}_{2} \leq \mathfrak{e}_{2}$

(d) $\mathfrak{r}_{\infty} \leq \min \left(\mathfrak{r}_{2}, \mathfrak{d}\right)$.

In Section 2 we prove the consistency of $\mathfrak{r}_{2}<\mathfrak{b}$.

In Section 3 we show that below $\mathfrak{r}_{\infty}$ one can properly extend an independent family of subsets of $\omega$ preserving a prescribed group of automorphisms. This is the relevance of $\mathfrak{r}_{\infty}$ to the behavior of homogeneous families under inclusion, which was the original motivation for the discovery of rules.

\section{Rules}

Definition 1.1. (1) A rule is a sequence $\vec{A}=\left(A_{n}, B_{n}: n \in \omega\right)$, where the sets $B_{n}$ are disjoint and finite, and for all $n, A_{n} \subseteq B_{n} \subseteq \omega$.

(2) We say that $X \in[\omega]^{\omega}$ follows the rule $\vec{A}$ if there are infinitely many $n$ with $X \cap B_{n}=A_{n}$; otherwise $X$ is said to avoid $\vec{A}$.

(3) For $k \in \omega$ we say that $\vec{A}$ is a $k$-rule if all sets $B_{n}$ have size $\leq k$. We say that $\vec{A}$ is a bounded rule if $\vec{A}$ is a $k$-rule for some $k$.

(4) More generally, for any function $f: \omega \rightarrow \omega$ we say that $\vec{A}$ is an $f$-rule if for all $n,\left|B_{n}\right| \leq f(n)$. We say that $f$ is a "slow" function if

$$
\sum_{n=0}^{\infty} 2^{-f(n)}=\infty
$$

and we say that $\vec{A}$ is a slow rule if it is an $f$-rule for some slow $f$.

Definition 1.2. (1) For $k \in \omega$ let $\mathfrak{r}_{k}:=\min \{|\mathfrak{R}|:$ there is no $X$ which follows all $k$-rules from $\mathfrak{R}\}$. (Similarly $\mathfrak{r}_{f}$, when $f: \omega \rightarrow \omega$.)

(2) Dually, let $\mathfrak{s}_{k}:=\min \{|\mathfrak{S}|:$ every $k$-rule is followed by some $X \in \mathfrak{S}\}$.

(3) We let $\mathfrak{r}_{\infty}=\min \{|\mathfrak{R}|$ : there is no $X$ which follows all bounded rules from $\mathfrak{R}\}$.

We remark that $2^{k}$ trivially bounds the least cardinality of a set of $k$-rules with the property that every real follows some rule in the set.

Recall that the "splitting" number $\mathfrak{s}$ and the "reaping" number $\mathfrak{r}$ are defined as follows:

Definition 1.3. If $s, X \in[\omega]^{\omega}$, then we say that $s$ "splits" $X$ if $s$ divides $X$ into two infinite parts, i.e., $s \cap X$ and $(\omega-s) \cap X$ are both infinite.

(1) $\mathfrak{s}:=\min \left\{|\mathfrak{S}|: \mathfrak{S} \subseteq[\omega]^{\omega}\right.$, every $X \in[\omega]^{\omega}$ is split by some $\left.s \in \mathfrak{S}\right\}$.

(2) $\mathfrak{r}:=\min \left\{|\mathfrak{R}|: \mathfrak{R} \subseteq[\omega]^{\omega}\right.$, there is no $X \in[\omega]^{\omega}$ which splits all $\left.r \in \mathfrak{R}\right\}$

Fact 1.4. $\cdots \leq \mathfrak{r}_{3} \leq \mathfrak{r}_{2} \leq \mathfrak{r}_{1}=\mathfrak{r}$, and $\mathfrak{s} \leq \mathfrak{s}_{2} \leq \mathfrak{s}_{3} \leq \cdots$. However, $\mathfrak{s}_{1}=2$, witnessed by $\mathfrak{S}=\{\emptyset, \omega\}$.

Theorem 1.5. (a) Let $(N, \in)$ be a model of $Z F C^{*}$ (a large enough fragment of $Z F C$ ). If a real $X$ follows all rules from $N$, then $X$ is Cohen over $N$. (Conversely, a Cohen real over $N$ follows all rules from $N$.) 
(b) If $X$ is random over $N$, then $X$ follows all slow rules from $N$ (so in particular, all bounded rules).

(c) $\max (\operatorname{cov}(\mathbb{K}), \operatorname{cov}(\mathbb{L})) \leq \mathfrak{r}_{\infty} \cdot(\operatorname{cov}(\mathbb{K})$ is the smallest number of first category sets needed to cover the real line. $\operatorname{cov}(\mathbb{L})$ is defined similarly using measure zero sets.)

Proof. (a): Assume that $X \subseteq \omega$ follows all rules from $N$. We claim that $\chi_{X}$, the characteristic function of $X$, is a Cohen real over $N$, that is, the set $\left\{\chi_{X}\lceil n: n \in \omega\}\right.$ is generic for the forcing notion $<\omega_{2}$.

To verify this claim, consider any nowhere dense tree $T \subseteq{ }^{<\omega_{2}} 2$ in $N$. We have to check that $\chi_{X}$ is not a branch of $T$.

Using the fact that $T$ is nowhere dense (and $T$ is in $N$ ) we can by induction (in $N$ !) find sequences $\left(n_{i}: i<\omega\right)$ and $\left(\eta_{i}: i<\omega\right)$ such that for all $i<\omega$ we have:

1. $n_{i}<n_{i+1}, \eta_{i} \in\left[n_{i}, n_{i+1}\right) 2$.

2. For all $\nu \in{ }^{n_{i}} 2, \nu \cup \eta_{i} \notin T$.

Now let $B_{i}:=\left[n_{i}, n_{i+1}\right), A_{i}=\left\{k: \eta_{i}(k)=1\right\}$. Our assumption tells us that $X$ follows the rule $\left(A_{i}, B_{i}: i \in \omega\right)$. So for some $i$ we have $X \cap B_{i}=A_{i}$, and hence $\chi_{X} \supseteq \eta_{i}$. Hence $\chi_{X}$ is not a branch of $T$.

This concludes the proof of (a).

The converse to (a) is obvious.

(b) is also easy: Let $X_{n}:=\left\{X: X \cap B_{n} \neq A_{n}\right\}$. For $n \neq m$, the sets $X_{n}$ and $X_{m}$ are independent (in the probabilistic sense), and $\mu\left(X_{n}\right)=1-2^{-f(n)}$, where $\mu$ is the Lebesgue measure on $\mathcal{P}(\omega) \simeq{ }^{\omega} 2$. Hence $\mu\left(\bigcap_{n>m} X_{n}\right)=\prod_{n>m}\left(1-2^{-f(n)}\right)=0$.

(c) follows from (a) and (b).

Theorem 1.6 (Shelah). For $k \geq 2, \mathfrak{r}_{k}=\mathfrak{r}_{2}$ (and similarly, $\mathfrak{s}_{2}=\mathfrak{s}_{k}$ ).

Proof. We will show that $\mathfrak{r}_{k}=\mathfrak{r}_{k+1}$ : Let $N_{0}$ be sufficiently closed (say, a model of $\mathrm{ZFC}^{*}$, but closed under some recursive functions is sufficient) of size $<\mathfrak{r}_{k}$; we have to show that there is a real that follows all $k+1$-rules from $N_{0}$.

We define a sequence $\left(N_{i}, C_{i}: i \leq k\right)$ such that $N_{i} \cup\left\{C_{i}\right\} \subseteq N_{i+1}$, each $N_{i}$ is sufficiently closed and of the same cardinality as $N_{0}$, and $C_{i}$ follows all $k$-rules from $N_{i}$.

Now let $C$ be the "average" of the $C_{i}: m \in C$ iff $m$ is in "most" of the $C_{i}$ 's, or formally:

$$
C:=\left\{m \in \omega:\left|\left\{i \leq k: m \in C_{i}\right\}\right|>(k+1) / 2\right\}
$$

Now we check that $C$ indeed follows all $k+1$-rules from $N_{0}$.

Let $\left(A_{n}, B_{n}: n \in \omega\right)$ be a $k+1$-rule in $N_{0}$. For $0 \leq i \leq k$ we let $\left(A_{n}^{i}, B_{n}^{i}: n \in \omega\right)$ be the $k$-rule obtained by removing the each $i$ th element of $B_{n}$. That is, letting $\left\{b_{n}^{0}, \ldots, b_{n}^{k}\right\}$ be the increasing enumeration of $B_{n}$ we let $B_{n}^{i}:=B_{n}-\left\{b_{n}^{i}\right\}, A_{n}^{i}:=$ $A_{n} \cap B_{n}^{i}$.

Let $E_{0}:=\omega$. For $0 \leq i \leq k$ let

$$
E_{i+1}:=\left\{n \in E_{i}: B_{n}^{i} \cap C_{i}=A_{n}^{i}\right\},
$$

i.e., $E_{i+1}$ is the set of indices on which $C_{i}$ follows the rule $\left(A_{n}^{i}, B_{n}^{i}: n \in E_{i}\right)$. Note that $E_{i} \in N_{i}$ and $C_{i} \in N_{i+1}$. By the choice of $C_{i}$ we know that each $E_{i+1}$ is infinite.

We conclude the proof by showing that for $n \in E_{k+1}$ we have $A_{n}=B_{n} \cap C$. Let $n \in E_{k+1}$ (so also $n \in E_{i}$ for all $i \leq k$ ), and $m \in B_{n}$. Say $m=b_{n}^{j}$. Then for $i \neq j$ we have $m \in B_{n}^{i}$, so $m \in A_{n}^{i} \Leftrightarrow m \in C_{i}$. 
Hence the cardinality of the set $\left\{i \leq k: m \in C_{i}\right\}$ is either in $\{0,1\}$ (iff $m \notin A_{n}$ ) or in $\{k, k+1\}$. In any case we get $m \in C$ iff $m \in A_{n}$. So $A_{n}=B_{n} \cap C$.

Theorem 1.7. $\mathfrak{r}_{\infty} \geq \min \left(\mathfrak{r}_{2}, \mathfrak{d}\right)$. In particular, if $\mathfrak{r}_{2} \leq \mathfrak{d}$, then $\mathfrak{r}_{\infty}=\mathfrak{r}_{2}$.

Proof. Let $N$ be a model of $\mathrm{ZFC}^{*}$ of cardinality $<\min \left(\mathfrak{r}_{2}, \mathfrak{d}\right)$. We will find a real $X$ following all bounded rules from $N$.

Define sequences $\left(N_{i}: i<\omega\right),\left(X_{i}: i<\omega\right)$ satisfying the following conditions:

1. $N_{0}=N$.

2. $N_{i}$ is a model of $\mathrm{ZFC}^{*}, N_{i-1} \cup\left\{X_{i}\right\} \subseteq N_{i}$.

3. $\left|N_{i}\right|=\left|N_{0}\right|$.

4. $X_{i}$ follows all $i$-rules (and hence also all $j$-rules for $j \leq i$ ) from $N_{i-1}$.

Let $N_{\omega}$ be a model of size $\left|N_{0}\right|$ containing $\left(N_{i}: i<\omega\right)$ and $\left(X_{i}: i<\omega\right)$. Since $\left|N_{\omega}\right|<\mathfrak{d}$, we can find a strictly increasing function $f$ that is not dominated by any function from $N_{\omega}$.

Define $X \subseteq \omega$ by requiring $X \cap(f(i-1), f(i)]=X_{i} \cap(f(i-1), f(i)]$. We claim that $X$ follows all bounded rules from $N$.

To complete the proof, consider an arbitrary $k$-rule $\left(A_{n}, B_{n}: n \in \omega\right)$ from $N$. We may assume $\min \bigcup_{n} B_{n}>f(k)$. We define sequences $\left(E_{i}: k \leq i<\omega\right)$ satisfying the following conditions for all $i \geq k$.

1. $\forall n \in E_{i} B_{n} \cap X_{i}=A_{n}$.

2. $E_{i} \in N_{i}$.

3. $E_{i+1} \subseteq E_{i}$.

We can carry out this construction, because $\left(A_{n}, B_{n}: n \in E_{i}\right)$ is a rule in $N_{i}$, so we just choose $E_{i+1}$ to witness that $X_{i+1}$ follows this rule.

Now let $n_{i}:=\min E_{i}$. Clearly the function $i \mapsto n_{i}$ is in $N_{\omega}$. So we can find infinitely many $j$ such that $f(j)>\max B_{n_{j}}$.

We claim that for each such $j, X \cap B_{n_{j}}=A_{n_{j}}$. For all $i \in[k, j]$ we have $n_{j} \in E_{i}$, so $X_{i} \cap B_{j}=A_{j}$. Note that $B_{j} \subseteq[f(k), f(j))$, so we also have $X \cap B_{j}=A_{j}$.

Problem 1.8. Is $\mathfrak{r}_{\infty}<\mathfrak{r}_{2}$ consistent?

We remark that in the random real model we have $\mathfrak{r}_{2}=\operatorname{cov}(\mathbb{L})=\mathfrak{c}=\mathfrak{r}_{\infty}, \mathfrak{d}=\aleph_{1}$. So one cannot hope to prove $\mathfrak{r}_{2} \leq \mathfrak{d}$.

We now consider the invariant that is dual to $\mathfrak{r}_{k}$, and we compare it with the well-known "evasion" number.

Definition 1.9. $(\pi, D)$ is a $k$-predictor, if $D$ is an infinite subset of $\omega, \pi=\left(\pi_{n}\right.$ : $n \in D), \pi_{n}$ a function from ${ }^{n} k$ to $k$.

We say that $f \in{ }^{\omega} k$ evades $(\pi, D)$ if there are infinitely many $\ell \in D$ such that $f(\ell) \neq \pi_{\ell}(f\lceil\ell)$.

$$
\mathfrak{e}_{k}:=\min \{|N|: \forall \pi \exists f \in N: f \text { evades } \pi\} .
$$

Brendle in [1] investigated these and other cardinal invariants and showed that all $\mathfrak{e}_{k}$ are equal to each other.

The following construction connects rules with predictors.

Definition 1.10. Let $R=\left(A_{n}, B_{n}: n \in \omega\right)$ be a 2-rule. Define a 2-predictor $\left(\pi_{R}, D_{R}\right)$ as follows: 
1. $D_{R}=\left\{\max B_{n}: n \in \omega\right\}$.

2. If $\ell=\max B_{n}$, and $\left|A_{n}\right|=1$, then $\pi_{\ell}(f)=f\left(\min B_{n}\right)$ for all $f \in{ }^{\ell} 2$. Otherwise, $\pi_{\ell}(f)=1-f\left(\min B_{n}\right)$.

Lemma 1.11. Let $X \subseteq \omega$. If $\chi_{X}$ evades $\pi_{R}$, then either $X$ or $\omega-X$ follows $R$.

Proof. Let $\ell_{n}:=\max B_{n}, i_{n}=\min \left(B_{n}\right)$ for all $n$.

$X$ evades $\pi_{R}$, so there are infinitely many $n$ such that $X\left(\ell_{n}\right) \neq \pi_{\ell_{n}}\left(X\left\lceil\ell_{n}\right)\right.$.

Case 1: There are infinitely many such $n$ where in addition $\left|A_{n}\right|=1$.

So for each such $n, X\left(\ell_{n}\right) \neq \pi_{\ell_{n}}\left(X \mid \ell_{n}\right)=X\left(i_{n}\right)$. So $X\left(\ell_{n}\right) \neq X\left(i_{n}\right)$, so $X \cap B_{n}$ must be either $A_{n}$ or $B_{n}-A_{n}$. One of the two alternatives holds infinitely often. Hence, either there are infinitely many $n$ such that $X \cap B_{n}=A_{n}$, or there are infinitely many $n$ such that $(\omega-X) \cap B_{n}=A_{n}$.

Case 2: There are infinitely many such $n$ with $X\left(\ell_{n}\right) \neq \pi_{\ell_{n}}\left(X \mid \ell_{n}\right)$, where in addition $\left|A_{n}\right|=2$, i.e., $A_{n}=B_{n}$. So for each such $n, X\left(\ell_{n}\right) \neq \pi_{\ell_{n}}\left(X \mid \ell_{n}\right)=1-X\left(i_{n}\right)$. So $X\left(\ell_{n}\right)=X\left(i_{n}\right)$, so $X \cap B_{n}$ must be either $B_{n}$ or $\emptyset$. One of the two alternatives holds infinitely often. So again we either get infinitely many $n$ such that $X \cap B_{n}=A_{n}$, or infinitely many $n$ such that $(\omega-X) \cap B_{n}=A_{n}$.

Case 3: For infinitely many $n$ as above we have $A_{n}=\emptyset$. Similar to the above.

Corollary 1.12. $\mathfrak{s}_{2} \leq \mathfrak{e}_{2}$.

Proof. Let $N$ be a model (of set theory) witnessing $\mathfrak{e}_{2}$, i.e., for every 2-predictor $\pi$ there is a function $f \in N$ evading $\pi$.

Let $R$ be any 2-rule. There is $X \in N$ evading $\pi_{R}$, so either $X$ or $\omega-X$ (both in $N$ ) follows $R$.

Remark 1.13. $\mathfrak{s} \leq \mathfrak{e}_{2}$ is known. Brendle showed that $\mathfrak{s}<\mathfrak{s}_{2}$ is consistent (unpublished).

\section{Consistency of $\mathfrak{r}_{2}<\mathfrak{r}$}

We show here in contrast to Theorem 1.6 that $\mathfrak{r}$ is not provably equal to $\mathfrak{r}_{2}$. Moreover, whereas $\mathfrak{b} \leq \mathfrak{r}$ is provable in ZFC (see [6] for a collection of results on cardinal invariants), we show that $\mathfrak{r}_{2}<\mathfrak{b}$ is consistent with ZFC.

The following definition is standard:

Definition 2.1. $\quad$ 1. $S$ is a slalom iff $\operatorname{dom}(S)=\omega$ and for all $n \in \omega, S(n)$ is a finite set of size $n$.

2. If $f$ is a function with $\operatorname{dom}(f)=\omega, S$ a slalom, then we say that $S$ captures $f$ iff $\forall^{\infty} n f(n) \in S(n)$.

3. Let $M \subseteq N$ be sets (typically: models of $\mathrm{ZFC}^{*}$ ). We say that $N$ has the Laver property over $M$ iff:

For every function $H \in{ }^{\omega} \omega \cap M$, for every function $f \in{ }^{\omega} \omega \cap N$

satisfying $f \leq H$ there is a slalom $S \in M$ that captures $f$.

4. A forcing notion $P$ has the Laver property iff $\Vdash_{P}$ " $V^{P}$ has the Laver property over $V . "$

Before we formulate the main lemma, we need the following easy claim:

Claim 2.2. Let $k>2^{n}$. If $X \subseteq{ }^{k} 2,|X|=n$, then there are $i<j$ in $k$ such that for all $f \in X, f(i)=f(j)$. 
Proof. For $i<j, f \in X$, define an equivalence relation $\sim_{f}$ by: $i \sim_{f} j \Longleftrightarrow f(i)=$ $f(j)$. Let $i \sim j$ iff $i \sim_{f} j$ for all $f$ in $X$. Since each $\sim_{f}$ has at most 2 equivalence classes, $\sim$ has at most $2^{n}$ classes, so there are $i \neq j, i \sim j$.

Lemma 2.3. Assume that $(N, \in)$ is a model of $Z F C^{*}$, and that $V$ has the Laver property over $N$.

Then every real avoids some 2-rule from $N$.

Proof. Let $a_{0}=0, a_{n+1}=a_{n}+2^{n}+1$. The sequence $\left(a_{n}: n \in \omega\right)$ is in $N$.

For any $X \in \mathcal{P}(\omega)$, we will find a rule in $N$ which $X$ does not follow.

Let $\chi_{X} \in{ }^{\omega} 2$ be the characteristic function of $X$. Define $X^{*}:=\left(\chi_{X}\left\lceil\left\lceil a_{n}, a_{n+1}\right)\right.\right.$ : $n \in \omega)$. Note that there are only $2^{2^{n}+1}$ many possibilities for $\chi_{X} \uparrow\left(a_{n}, a_{n+1}\right)$.

Since $V$ has the Laver property over $N$ there is a sequence $\vec{S}=\left(S_{n}: n \in \omega\right) \in N$, $S_{n} \subseteq\left[a_{n}, a_{n+1}\right) 2,\left|S_{n}\right| \leq n$, and for all $n>0, \chi_{X}\left\lceil\left[a_{n}, a_{n+1}\right) \in S(n)\right.$. By the above claim we can find $i_{n}<j_{n}$ in $\left[a_{n}, a_{n+1}\right)$ such that for all $z \in S(n), z\left(i_{n}\right)=z\left(j_{n}\right)$. Since the sequence $\vec{S}$ is in $N$, we can find such a sequence $\left(i_{n}, j_{n}: n<\omega\right)$ in $N$.

Define a 2-rule $\left(A_{n}, B_{n}: n \in \omega\right) \in N$ by $A_{n}=\left\{i_{n}\right\}, B_{n}=\left\{i_{n}, j_{n}\right\}$. Since $i_{n} \in X$ iff $j_{n} \in X, X$ does not follow this rule.

Lemma 2.4. (a) Let $\bar{P}=\left(P_{i}, Q_{i}: i<\omega_{2}\right)$ be a countable support iteration of proper forcing notions such that for each $i$ we have $\Vdash_{i}$ " $Q_{i}$ has the Laver property." Then $P_{\omega_{2}}$, the countable support limit of $\bar{P}$, also has the Laver property.

(b) Laver forcing is proper and has the Laver property.

(c) Laver forcing adds a real that dominates all reals from the ground model.

Proof. These facts are well known and (at least for the case where each $Q_{i}$ is Laver forcing) appear implicitly or explicitly in Laver's paper [4].

Conclusion 2.5. Let $P_{\omega_{2}}$ be the limit of a countable support iteration of Laver forcing over a model $V_{0}$ of GCH. Then $\Vdash_{P_{\omega_{2}}} \mathfrak{b}=\mathfrak{r}=\omega_{2}$ and $\mathfrak{r}_{2}=\omega_{1}$.

Proof. Let $V_{\omega_{2}}=V^{P_{\omega_{2}}} . V_{\omega_{2}} \models \mathfrak{b}=\omega_{2}$ is well known. (Let $f_{i}$ be the real added by the $i$ th Laver forcing; then $\left(f_{i}: i<\omega_{2}\right)$ is a strictly increasing and cofinal sequence in ${ }^{\omega} \omega$.)

By 2.4, $V_{\omega_{2}}$ has the Laver property over $V_{0}$. Hence, by 2.3, every real avoids some rule from $V_{0}$. So $\mathfrak{r}_{2} \leq\left|{ }^{\omega} \omega \cap V_{0}\right|=\aleph_{1}$.

\section{Application to independent families}

A family $\mathcal{F} \subseteq \mathcal{P}(\omega)$ of subsets of $\omega$ is independent iff it generates a free boolean algebra in $\mathcal{P}(\omega) /$ fin. Equivalently, for any two disjoint finite subsets of $\mathcal{F}$, the intersection of all members in the first set with all complements of members in the second set is infinite.

The following is an example of an independent family of size continuum over a countable set: $\left\{A_{r}: r \in \mathbb{R}\right\}$ where $A_{r}=\{p \in \mathbb{Z}[X]: p(r)>0\}$.

A family $\mathcal{F} \subseteq \mathcal{P}(\omega)$ is dense iff for any two finite disjoint subsets of $\omega$ there are infinitely many members of $\mathcal{F}$ that contain the first set and are disjoint to the second.

An interesting (proper) subclass of the class of dense independent families over $\omega$ is the class of homogeneous families, which was introduced in [2]. Its study was continued in [3]. 
While every dense independent family is contained in a maximal dense independent family, this is not obvious (and perhaps false) for homogeneous families. The existence, even the consistency, of a maximal homogeneous family over $\omega$ is still open. In particular, an increasing union of homogeneous families need not be homogeneous.

In the study of extendibility of homogeneous families, the following notion is fundamental: Let $G \subseteq$ Aut $\mathcal{F}$. We define $(\mathcal{F}, G) \leq\left(\mathcal{F}^{\prime}, G^{\prime}\right)$ iff $\mathcal{F} \subseteq \mathcal{F}^{\prime}, G \subseteq G^{\prime} \subseteq$ Aut $\mathcal{F}^{\prime}$. The usefulness of $\leq$ is that unions of suitable $\leq$ chains are homogeneous (see [3] for a detailed account of direct limits in the category of homogeneous families).

We show now that below $\mathfrak{r}_{\infty}$ one can get proper $\leq$-extensions of independent families. This was our original motivation for discovering $\mathfrak{r}_{\infty}$.

Theorem 3.1. Suppose $G \subseteq$ Aut $\mathcal{F}, \mathcal{F} \subseteq \mathcal{P}(\omega)$ is dense independent and $|\mathcal{F}|+$ $|G|<\mathfrak{r}_{\infty}$. Then there exists $\mathcal{F}^{\prime} \supsetneqq \mathcal{F}$ such that $(\mathcal{F}, G) \leq\left(\mathcal{F}^{\prime}, G\right)$.

Proof. Suppose that $G \subseteq$ Aut $\mathcal{F}, \mathcal{F}$ is dense independent and $|G|+|\mathcal{F}|<r_{\infty}$. We shall find a real $X \subseteq \omega$ such that $X \notin \mathcal{F}$ and $\mathcal{F} \cup G[X]$ is independent, where $G[X]$ is the orbit of $X$ under $G$. This will suffice, since clearly $G \subseteq \operatorname{Aut}(\mathcal{F} \cup G[X])$ for any real $X$.

It is a priori unclear why such $X$ should exist. If for example there is some $\sigma \in G$ with finite support, then for no $X \subseteq \omega$ is even the orbit $G[X]$ itself independent. However, the following lemma takes care of this. Let $\operatorname{supp}(\sigma)=\{n \in \omega: \sigma(n) \neq n\}$ for a permutation $\sigma \in \operatorname{Sym} \omega$.

Lemma 3.2. Suppose that $\mathcal{F}$ is dense independent and $\sigma \in$ Aut $\mathcal{F}$ is not the identity. Then there are distinct sets $C_{n} \in \mathcal{F}$ such that for all $n, C_{2 n}-C_{2 n+1} \subseteq \operatorname{supp} \sigma$.

Remark 3.3. In particular, the supports of non-identity automorphisms have the finite intersection property and hence generate a filter. This is the "strong Mekler condition" for Aut $\mathcal{F}$ (see [5]).

Proof. Fix $k^{*} \in \omega$ for which $\sigma\left(k^{*}\right) \neq k^{*}$. Find $C_{2 n}, C_{2 n+1}$ by induction on $n$. Suppose $C_{m}$ is chosen for $m<2 n$. By density, there are infinitely many $C \in \mathcal{F}$ for which $k^{*} \in C, \sigma\left(k^{*}\right) \notin C$. Choose some such $C$ so that neither $C$ nor $\sigma[C]$ are among $\left\{C_{m}: m<2 n\right\}$. Let $C_{2 n}=C$ and $C_{2 n+1}=\sigma[C]$. Since $k^{*} \in C_{2 n}-C_{2 n+1}$, those sets are indeed distinct.

We claim that $C_{2 n}-C_{2 n+1} \subseteq \operatorname{supp}(\sigma)$. Indeed, for any $k \in C_{2 n}-C_{2 n+1}$ we have $\sigma(k) \in C_{2 n+1}$ but $k \notin C_{2 n+1}$, so $k \neq \sigma(k)$.

We now continue the proof of Theorem 3.1. Let $M$ be a transitive model of ZFC* of cardinality $<\mathfrak{r}_{\infty}$ such that $\mathcal{F}, G \in M$ and $G \subseteq M, \mathcal{F} \subseteq M$. Let $X$ be a real that follows all bounded rules from $M$. Clearly, $X \notin M$, and therefore $X \notin \mathcal{F}$.

We need to show that every boolean combination over $\mathcal{F} \cup G[X]$ is infinite. Suppose that

$$
D:=A \cap \sigma_{0}[X] \cap \cdots \cap \sigma_{n-1}[X] \cap\left(\omega-\sigma_{n}[X]\right) \cap \cdots \cap\left(\omega-\sigma_{m-1}[X]\right)
$$

is a boolean combination over $\mathcal{F} \cup G[X]$, where $\sigma_{i} \in G$ for $i<m$, and $A$ is some boolean combination over $\mathcal{F}$. Clearly, $A \in M$.

Set $N=\left(\begin{array}{c}m \\ 2\end{array}\right)$ and let $\left(\tau_{i}: i<N\right)$ be a list of all $\sigma_{k} \circ \sigma_{\ell}^{-1}$ for $k<\ell<m$. By induction find a sequence $\left(C_{0}, \ldots, C_{2 n+1}\right)$ of $2 N+2$ many distinct sets such that no $C_{k}$ participates in $D$ and such that $C_{2 k}-C_{2 k+1} \subseteq \operatorname{supp} \tau_{k} . C_{2 k}$ and $C_{2 k+1}$ are constructed in the $k$-th step by using Lemma 3.2. 
Since all the $C_{k}$ are distinct, the intersection $E=A \cap \bigcap_{k<N} C_{2 k}-\bigcup_{k<N} C_{2 k+1}$ is infinite. Clearly $E$ belongs to $M$. Define by induction an $m$-rule $\left(A_{n}, B_{n}: n<\omega\right)$ as follows: suppose $\left(A_{k}, B_{k}: k \leq n\right)$ are defined. Find a point $j_{n} \in E$ such that $B=\left\{\sigma_{k}^{-1}\left(j_{n}\right): k<m\right\}$ is disjoint from $\bigcup_{\ell \leq n} B_{\ell}$ and $j_{n} \notin \bigcup_{\ell \leq n} B_{\ell}$. Let $B_{n+1}$ be $B$ and let $A_{n+1}=\left\{\sigma_{\ell}^{-1}\left(j_{n}\right): \ell<n\right\}$.

The rule we defined obviously belongs to $M$. Since $X$ satisfies all bounded rules from $M$, there are infinitely many $n$ for which $X \cap B_{n}=A_{n}$. For each such $n$, $X_{n} \in D$.

\section{REFERENCES}

[1] Jörg Brendle. Evasion and prediction-the Specker phenomenon and Gross spaces. Forum Math., 7(5):513-541, 1995. MR 96i:03042

[2] Martin Goldstern, Rami Grossberg, and Menachem Kojman. Infinite Homogeneous Bipartite Graphs With Unequal Sides. Discrete Mathematics, 149:69-82, 1996. MR 97a:05102

[3] Menachem Kojman and Saharon Shelah. Homogeneous families and their automorphism groups. Journal of the London Mathematical Society, 52:303-317, 1995.

[4] R. Laver. On the consistency of Borel's conjecture. Acta Math., 137:151-169, 1976. MR 54:10019

[5] J. K. Truss. Embeddings of infinite permutation groups. In Proceedings of groups - St Andrews 1985, volume 121 of London Math. Soc. Lecture Series, pages 335-351. Cambridge University Press, 1986. MR 89d:20002

[6] J.E. Vaughan. Small uncountable cardinals in topology. In Open problems in topology, pages 217-218. Elsvier Science Publishers, B.V. North Holland, 1990. ed. van Mill, J. and Reed, G.M.

Institut für Algebra, Technische Universität, Wiedner Hauptstrasse 8-10/118.2, A1040 Wien, Austria

E-mail address: Martin.Goldstern@tuwien.ac.at

Department of Mathematics, Ben-Gurion University of the Negev, POB 653. BeerSHEVA 84105, ISRAEL

E-mail address: kojman@math.bgu.ac.il

Current address: Department of Mathematics, Carnegie Mellon University, 5000 Forbes Ave, Pittsburgh, Pennsylvania 15213 